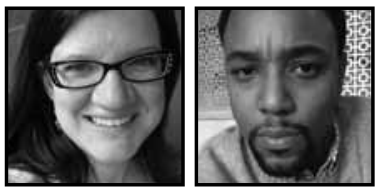

\title{
Supporting Climate Science Research With 21st Century Technologies and a Virtual Student Confer- ence for Upper Elementary to High School Students
}

\author{
Julie S. Malmberg, The GLOBE Program \\ Keith E. Maull, University of Colorado
}

\section{ABSTRACT}

Engaging young science learners today requires a plethora of tools that oftentimes leverages technology in novel ways. This paper describes the use of several 21st century technologies to engage science learners in locally relevant climate science research projects and the presentation of these projects in an entirely online virtual student conference. Case studies demonstrating the use of and effectiveness of 21st century technologies and GLOBE protocols are also included. Through technology, students were able to find out more about distant locations and their own environments, talk to scientists, and make studying climate science personally relevant. Finally, the implementation and structure of the GLOBE Virtual Student Conference is described.

\section{Introduction}

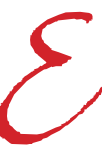

ngaging science learners today is challenging and there is a continued push to develop Science Technology Engineering and Math (STEM) curricula that meaningfully integrate technology into as many curricular layers as possible (National Research Council, 2012). Of the many challenges teaching science, engaging learners oftentimes requires deepening the connection to the science through tangible concepts that address contemporary issues demonstrating the 
scientific phenomenon at hand (Hodson, 2003). Doing this requires tools that bring the learner successfully into the science, and climatology is an excellent vehicle to do that in many important ways. First, there are many open, freely available datasets that can be utilized to examine long-term climate trends. These may provide excellent opportunities for learners to immerse themselves in a particular aspect of a problem of interest. Second, since both the data and the problems being studied are often of high complexity, breaking them down into smaller, more manageable problems provides excellent opportunities to develop self-contained research questions that can be supported with effective pedagogy and collaboration (Fleming, 2010). Finally, many climate phenomenon can be observed directly, allowing young learners to get first-hand access to the scientific processes at hand as well as empirical data collection that can be experienced as much as it can be analyzed.

Another key challenge in the classroom is bringing technology to the learner, in ways that they are easily able to make productive use of technology to solve problems. With the multitude of technology platforms available (e.g., social media, online educational tools, online data repositories), and the increasingly distributed nature of communications, this paper will present a use of YouTube, Adobe Connect and Skype online video communications to provide learners direct control over technology while sharing their work with a distributed network of peers working on similar scientific concepts. This achieves two goals: the first technological, the second, pedagogical. On the technological side, students learn to increase their mastery of tools they may already be aware of, but may or may not already be using in the context of their own educational endeavors. While the consumption of technology remains high among learners today (Lenhart, Purcell, Smith, \& Zickuhr, 2010), continuing to utilize these technologies in productive and creative ways remains a goal, especially within educational contexts, where other distractions may be reducing students' educational interests around technology use. On the pedagogical side, as learners engaged with other learners doing the same science in geographically and climatically diverse areas, they are able to connect the science concepts being learned in their own local environments with those across geography, and through carefully constructed exercises and discussions, reinforce the science even further. Thus, the central research question of this paper peers into both of these goals by positing: What are engaging ways to use collaborative, 21 st century technologies to encourage students to learn about and share their understandings of climate science?

None of the gains in science understanding and technology use can be effective without appropriate teacher training and support (Fleming, 2010), particularly when considering the inquiry-based instruction practices that are becoming 
commonplace in science curricula today. While most science teachers are receiving some form of ongoing professional development, several dimensions of effective professional development in inquiry-based instruction have been identified including (but not limited to) extended support, authentic experience, reflection, content knowledge, enhanced knowledge, and change in teacher beliefs (Capps, Crawford, \& Constas, 2012). Furthermore, Garet, Porter, Desimone, Birman, and Yoon (2001) and Penuel, Fishman, Yamaguchi, and Gallagher (2007) suggest that effective inquirybased professional development focus on content knowledge and integration of activities into the classroom, while Darling-Hammond and McLaughlin (1995) and Loucks-Horsley, Hewson, Love, and Stiles (1998) suggest that professional development foster communities that value and support continuous learning, among other things. Though this paper will only briefly describe the teacher supports used within this project, several important features of the teacher training that was provided are: (1) teachers engaged in an online community of professional peers where they were in contact regularly, (2) participating teachers were encouraged to engage in bi-weekly webinars that provided a professional community platform for them to extend their content knowledge and share their experiences with one another, and (3) a focused curriculum was developed that centered around climate science that provided access to relevant content knowledge and sustained conceptual exposure that helped teachers develop throughout the course of the school year.

This paper is divided into three segments. The first segment will describe The GLOBE Program, its purpose, benefits, and use within this project. Furthermore, the first segment will lay out the rationale of the climate change curriculum used in this project-From Learning to Research (L2R)—and its intended goals and challenges. The second segment will describe three case studies of the use of the GLOBE From Learning to Research program and the resulting use of technology to engage learners in the production of outcomes resulting from applying science concepts directly from the curriculum. The third segment of this paper will reflect on the successes of the case studies and suggest a few ways that technology can be applied within the classroom to improve student engagement and learning. The final segment will reflect on and describe the implementation of the GLOBE Virtual Student Conference and how students can share and view research projects regardless of physical location. 


\section{The GLOBE Program and From Learning to Research Curriculum}

\section{The GLOBE Program}

The GLOBE (Global Learning and Observations to Benefit the Environment) Program, sponsored by NASA, NOAA, and NSF, is a worldwide science and education program designed to encourage and support educators, students, and scientists in collaborative, inquiry-based Earth system scientific investigations. With over 112 countries, 24,000 schools and 1.5 million students having participated in the GLOBE program, the GLOBE database has over 23 million measurements that are being used in inquiry-based classroom lectures, discussions, and projects. With such a large network of participants, The GLOBE Program provides unique learning opportunities and collaborations among schools, teachers, students, and scientists.

\section{From Learning to Research}

One program that supports GLOBE's efforts is the From Learning To Research (L2R) program. Designed to help engage learners in the scientific process, while at the same time develop their knowledge and understanding of Earth systems processes, the L2R program is focused on three areas. The first is to provide the necessary supports and curriculum to teachers to effectively engage their learners in climate science concepts. The second is to expose students to climate science research with experts to facilitate closing the gap between climate science concepts and real-world scientific applicability of those concepts. This is primarily done by providing students access to climate science experts (meteorologists, climatologists, and other field experts) at various points through the curriculum. The third is to utilize 21st century technologies to encourage collaboration, distributed learning, and to facilitate building connections between students' local climate phenomenon and those of their geographically distributed collaborators. By actively engaging learners in scientific concepts and inquiry-driven activities, while also allowing students to gain access to climate scientists, students begin to learn how the science concepts they are learning are directly applied to solve problems that they may be observing first-hand in their classroom learning and data collection activities, while also engaging in the actual science around their learning materials. Furthermore, they are able to see how local climate phenomenon, supported by data collection and scientific analyses, are similar (or different) from those of their distributed peers. 


\section{From Learning to Research: Goals}

Funded as an NSF Innovative Technology Experiences for Students and Teachers (ITEST) project, the L2R project seeks to develop a model for teacherstudent-scientist collaborations leveraging 21st century technologies. The L2R team consists of educators (both former K-12 teachers and professors in STEM education), technology facilitators, researchers in educational communication, and technology and physical scientists. The primary goals of $L 2 R$ are:

1. To provide teachers with the knowledge, skills, strategies, and confidence to engage middle and secondary students in authentic Earth System Science research on local, regional, and global scales

2. To expose teachers and students to careers in the Earth Sciences

3. To allow students to experience Earth System Science research by interacting with scientists from around the world

\section{From Learning to Research: Implementation}

In order to develop a successful collaboration model using 21st century technologies, teachers were recruited from geographically diverse areas. These included rural areas (some of the represented towns had either no or one stoplight), suburban and urban areas. Teachers were also recruited from diverse socioeconomic communities ranging from inner-city public schools to private schools. The 75 recruited teachers represented 22 states plus Puerto Rico and taught over 5,500 students in grades 5-12, including traditional schools as well as alternative schools. The teachers represented demographically diverse school settings and taught mainly science courses, including Advanced Placement Environmental Science, Earth Science, Biology, and general science, but some of the teachers taught math, technology, or geography. It was also preferred that teachers had three or more years of teaching experience to improve the stability of the project goals. Furthermore, in order to participate, teachers had to get a letter of support signed by their administrators. 


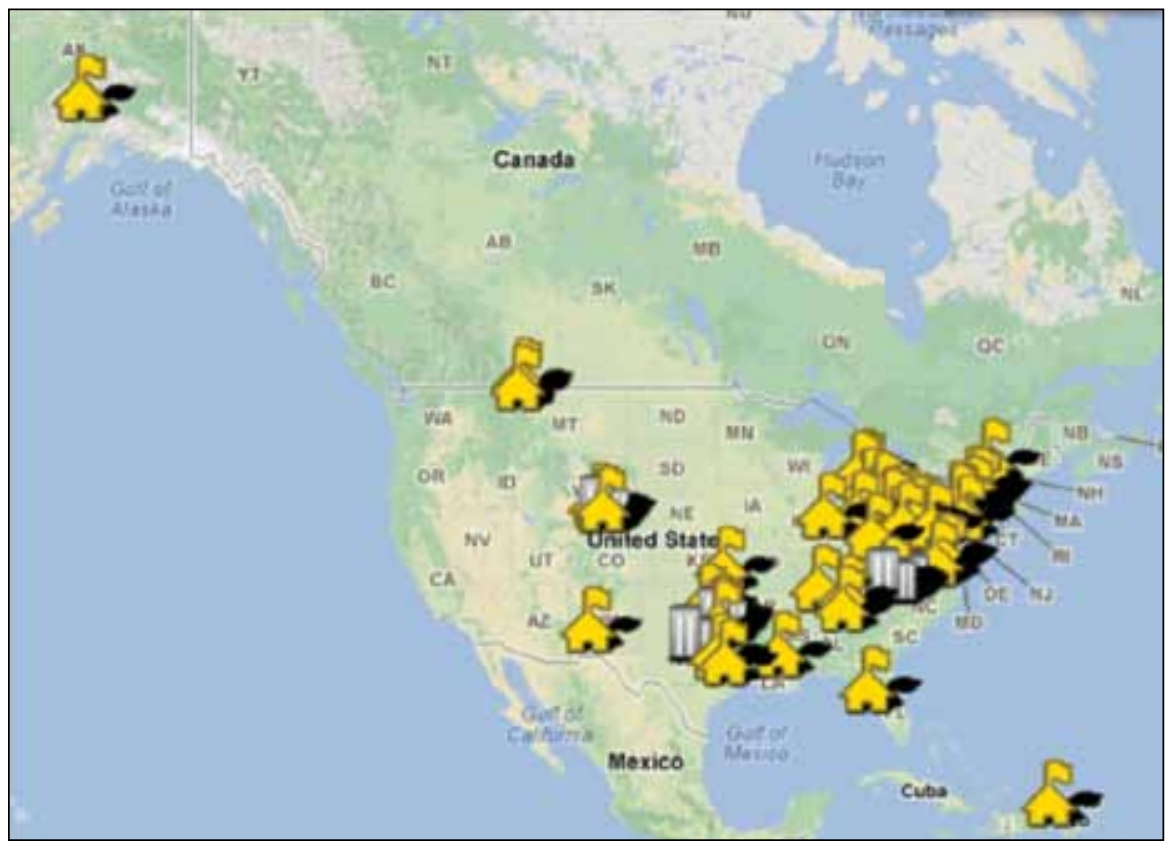

Fig. 1: A map showing geographic distribution of teachers involved in $L 2 R$

The outcome of participating in L2R is a student research project about a locally relevant climate or environmental issue while using 21 st century technologies and collaboration skills. To achieve this, the teachers participated in extensive professional development throughout the entire school year. This started with a weeklong summer institute held in Tyler, Texas in 2011 and Boulder, Colorado in 2012. The summer institute included presentations about project-based and inquiry-based learning, Next Generation Science Standards, a climate science overview, climate and climate change education, misconceptions and controversies in climate science, teaching in the 21st century (including skills and technologies), and two days of fieldwork. The teachers left the summer institute with concrete plans for collaboration, uses of technology, and a research project.

During the school year, teachers participated in webinars every two weeks, fall and spring status updates, and teacher updates on the student research projects. The webinars consisted of general announcements and interesting finds (e.g., useful technology tools or web pages), project updates, and career speakers from a variety of STEM fields. Each portion of the webinar was relatively short (10-12 minutes in length), so teachers shared the career speaker videos with their students in order to expose the students to many career options in STEM fields. Additionally, students 
communicated with other classrooms, the L2R staff and scientists via Skype, Adobe Connect, and email. Examples of student-to-student collaborations included sharing how to use a particular scientific instrument, updates about the class projects via Adobe Connect, and Q\&A times about the schools' local communities and climate. Students communicated with scientists via Skype, webinars, and email. Additionally, after the students watched the Adobe Connect recordings of STEM career speakers, the teachers and students emailed or posted questions to a discussion forum hosted on http://www.globe.gov.

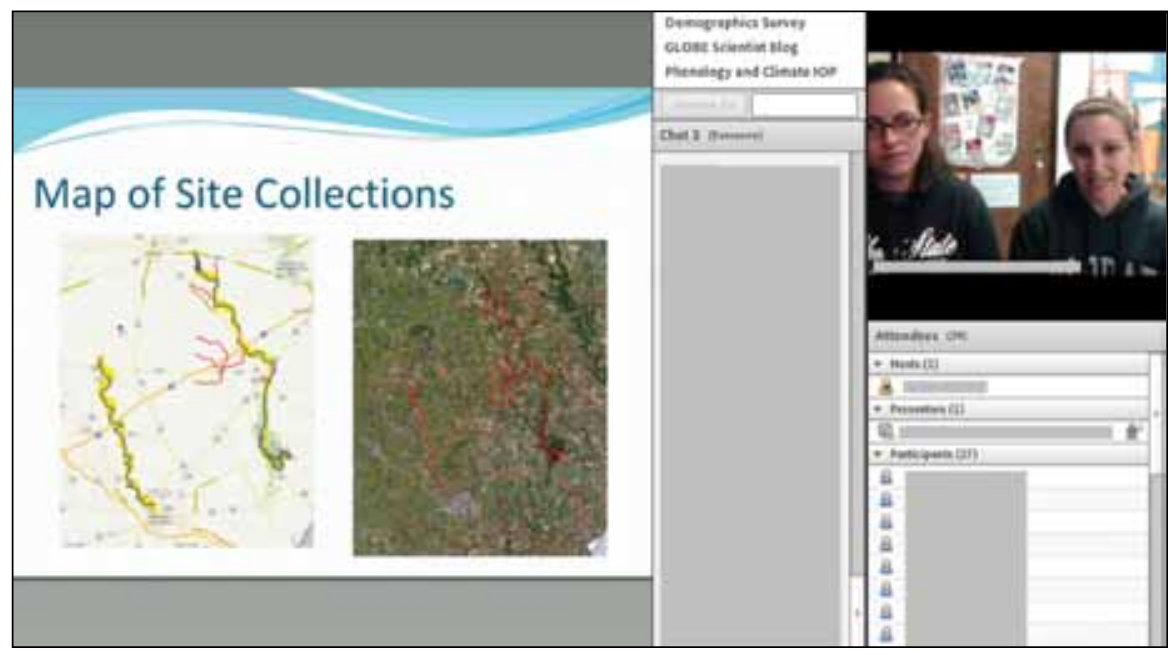

Fig. 2: An example of a project update via Adobe Connect. The presentation area is on the left-this can show PowerPoints, Prezis, web pages, or other documents, useful links and a chat window in the middle, and a webcam and participants on the right.

\section{L2R Project Case Studies}

Even with the training, teachers' use of technology in the classroom may vary greatly, and often with time and experience, teachers and students alike can get into effective and productive patterns of technology use. With the L2R project, two technologies, Skype and video recording, were widely encouraged to facilitate communication and collaboration. In this section, we will highlight three cases that demonstrate how these technologies helped students and teachers engage in climate science research across geographic boundaries, to enable deep learning experiences that would otherwise not be possible. We will also highlight the use of video technology through the "Student Virtual Conference" (described in more detail in the next 
section), and show how students became more engaged and excited about the science, through the connections they formed with others via these technologies. Note that any identifying information has been changed.

\section{Case I: Martha}

Martha is from a rural, politically conservative US town and had been a science teacher at a middle school for over a decade. She was very hesitant about teaching climate and climate change due to personal disbelief and concerns about negative responses from her community. She was encouraged by her administrators to participate in L2R, but during the first half of the year, Martha was not engaged in the program. However, after further push from her administrator, Martha began working more closely with L2R team member Amelia to explore potential locally relevant climate projects. Martha ended up choosing to work on phenology (the study of living organisms' responses to seasonal changes in their environment) in her area and agreed upon applying the green-up protocol. This GLOBE protocol is a relatively simple and inexpensive way to study the environment during which students monitor leaf buds in the spring. Her students selected several trees and started monitoring these trees for budburst and green-up. Martha, who had very little experience with climate science curricula, also worked closely with GLOBE staff to arrange Skype chat sessions between her students and climate scientists from different regions around the world. In these sessions, the scientists guided the students' research question, data collection and analysis, and presentation activities.

The students were so enthusiastic about studying phenology and climate, and talking to students and scientists in distant locations, that Martha became much more engaged in L2R and her students started investigating other research areas using GLOBE protocols in their community. These included looking at water quality (water temperature and transparency, dissolved oxygen, $\mathrm{pH}$, nitrates, freshwater macroinvertebrates) near a poultry farm, investigating how their collected precipitation data compared to normals (it was a drought year), and looking at long-term air temperature datasets.

Martha's students presented their research project at the 2012 Virtual Student Conference and were active participants in the online conference activities, asking other students questions about their projects and inquiring about the science that emerged from others' projects. At the conclusion of the first year of $L 2 R$, not only did Martha and her students expand their science knowledge and technology use, but several students have also gained international recognition from their work 
since the completion of their project. One of the more powerful aspects of the $L 2 R$ experience is summarized by one of Martha's students who said, "...it's been really interesting getting to be a part of it...it's neat to think that students in a small town of about 1,300 people get to interact with people from around the world because of a research project." Some of Martha's students continue to collaborate with other students and scientists from around the world.

Using technology, Martha and her students were able to explore opportunities that would otherwise be unavailable to them. They had access to some of the forefront scientists in the field and were able to see what other students around the country were exploring. Martha may not have had the skillset or confidence to study phenology and climate on her own, but with the support of other schools, scientists, and L2R staff, she was able to bring new opportunities to her students.

\section{Case II: Jack}

Jack, a 5th grade teacher in a rural US town, had been teaching for over 15 years. He had been involved with The GLOBE Program for over a decade and consistently sought out unique and engaging science opportunities (including climate science) for his students as well as professional development workshops and experiences for his own enrichment. When he saw the call for L2R recruitment, he immediately applied, and while Jack's students were already very active in collecting and analyzing data for local climate science projects, the technology component was both new and intriguing for Jack and his students. During the school year, Jack and his students started Skyping with students and scientists from around the world to share and learn more about their local environments. Through these Skype sessions Jack's students began to develop personal relationships and even began referring to other students they had been collaborating with as their "friends."

For the online Virtual Student Conference, Jack's students submitted a dramatic video rendition of their research project covering all elements of the scientific method. Most notably, Jack's students realized that not all research questions end up with definitive answers - an insight that even much older students struggle to realize and cope with. Jack and his students were later invited to participate in an international science competition to further present their research, which received both formal recognition and praise from the judges and other competition participants.

To show the importance of how distributed communications technologies helped his students to learn more science and gain valuable and authentic experiences, Jack pointed out in an interview: 
One of my favorite examples of how my students gained better conceptual understanding is the connection that became obvious with other schools in the USA as well as in the world. Quite often I would hear my students refer to other GLOBE schools as their friends. Finding and comparing surface temperature, snow, cloud data from schools in a different hemisphere, and viewing land cover photos from other locations, has helped my students to begin to understand the value in working together as a world-wide community to address climate and environmental issues.

Clearly, with these technologies available to Jack's students, building the connections between the scientific observations of their local climate and those of their online distributed peers, the importance and relevance of the science they were learning became much more meaningful.

\section{Case III: Melody}

Melody, a middle school science teacher in a geographically isolated location in the US, had been teaching for over 20 years, after switching careers from a corporate job earlier in her work history. Melody was concerned because her students were isolated from other regions of the country and worried that they would not become "citizens of the world." Many of the families in this location treated the area as a temporary home (even though they had lived there for ten years or more) and did not treat this area like a place they might stay for generations. The students were indifferent about recycling, keeping their areas clean, and embracing the local ecological features.

When she saw the announcement for the L2R professional development training that included collaboration via technology, Melody thought this would be an ideal opportunity for her students. After the summer teacher training, Melody started actively pursuing Skype relationships with schools around the world. Melody tapped into GLOBE's international database and contacted GLOBE schools from different regions, but due to the extreme isolation of her school, the Internet connection was substantially degraded for the bandwidth required to meaningfully participate. She consequently purchased a wireless hot spot to improve conductivity for her students, which then allowed her students to begin Skyping weekly with students from quite a few countries. Melody would even host lock-ins at the school to allow students to Skype very late at night with schools from distant locations at convenient times for those students. 
After Skyping with others from a variety of locations, Melody's students became interested in improving their local community. Her students began to take greater notice of some of the unique ecological features of their hometown and pursued conversations with scientists specializing in these features. For their $L 2 R$ research project, the students explored ways to improve their environment through a revitalization project while carefully documenting their progress. By using GLOBE protocols to collect and visualize their data, the students were able to see how modifying aspects of their environment helped to improve water quality. For the Virtual Student Conference, Melody's students showed before and after pictures of their community and explained how their research led to them improving their local environment.

Through technology, Melody's students not only learned about other environments, but also began to appreciate their own environment. And, even though Melody's students were in a very isolated location, they were able to connect with schools from a wide variety of locations. Rather than just reading about a distant location in a textbook, Melody's students were able to virtually see and discuss far-off environments with the students who lived in these locations.
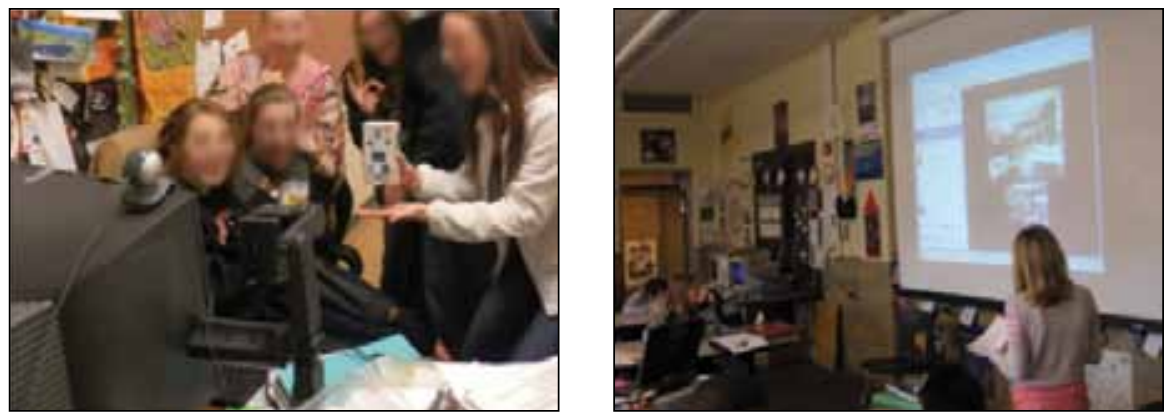

Fig. 3: Students showing their scientific instruments during a Skype session

\section{Synthesis}

These three case studies illustrate how 21st century technologies allowed students to learn about their environments in new ways. While collaboration with geographically distant scientists, teachers, and students was possible before Skyping, webinars, and social web pages, these advancements have made collaboration much more accessible for teachers-particularly since all the schools were doing GLOBE protocols for data collection, which are consistent regardless of location. Martha's students got to learn about a topic that might not have been otherwise taught. 
And, they were able to communicate with the scientists currently researching that science topic. Jack's students were able to compare and discuss their data with other schools' data. The students developed personal relationships with students in distant locations and were able to see how their research was important. Finally, through technology, Melody's students learned to appreciate the uniqueness of their own environment while learning about other cultures and environments as well. While we only presented three case studies, each teacher involved in L2R and his or her students were able to make connections, explore new topics, and learn more about the world via 21st century technologies.

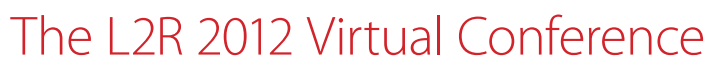

The culminating event of $L 2 R$ was for students to participate in the GLOBE Virtual Student Conference. Due to geographic, financial, and time constraints, a virtual conference was the best way for students to share their research with other classrooms. Despite these constraints, video technology was chosen as the primary tool for conducting the conference for several reasons. First, students were able to show details of their research projects, and in some cases this allowed for deeper capture and expression of the local qualities of their climate. Second, while students may already be familiar with online video through their own personal and academic use, they may not have used video to produce anything meaningful for the purposes of sharing with others, and more importantly, in the context of their own learning. Not only did this allow students to become full participants in their own projects, but also provided exposure to the ways technologies can be used to capture, record, and share their own educational knowledge and understanding with others. Finally, and perhaps more importantly, video technologies allow students to see firsthand the experiences of others engaging in activities similar to their own. With this medium, students are able to more intimately connect with the ideas (and science) of their peers. Also, with a push towards a more environmentally conscious world, a virtual conference has the added benefit of being "greener" than an in-person conference.

\section{Virtual Conference: Structure and Implementation}

To closely emulate the authentic structure of academic scientific conferences, students were asked to submit an abstract, a video documenting their local climate project, and a science report. The video portion was left open-ended, allowing students to create their video presentation in any style they liked. This yielded a variety 
of creative submission styles from narrated PowerPoints, videoblog-style reports, videos of the students in front of their science posters, to songs about the student projects. All the videos submitted were shared in two ways. First, they were uploaded to YouTube and embedded into a YouTube "channel," and second, for teachers that had school-imposed restricted access to YouTube, a video was also made available via download from a GLOBE server. YouTube was chosen primarily because of the readily available access to YouTube by the majority of the teachers, as well as the ease with which it allows videos to be shared, embedded, and viewed. Once submitted, the student projects were arranged into "rooms" (child pages of the conference web page) based upon themes, which included general atmosphere and climate, hydrology and climate, phenology and climate, and land cover and climate. Student projects were assigned a number and could be cross-listed in more than one theme.

The first GLOBE Virtual Student Conference was "open" for one day. During this day, teachers and students were encouraged to watch the videos, read the abstracts, and ask the other students questions about their projects via a commenting tool on the web page. GLOBE Scientists and community members also asked questions and made comments. Several of the schools spent significant portions of the school day watching the videos and invited administrators and other classes to watch with them. Students also Skyped other L2R schools and watched and discussed the projects together. The students answered the questions and responded to the comments during this virtual conference day. Since not all students were able to log in to the virtual conference on the same day, teachers and students were encouraged to check their projects for questions and comments for the following few weeks.

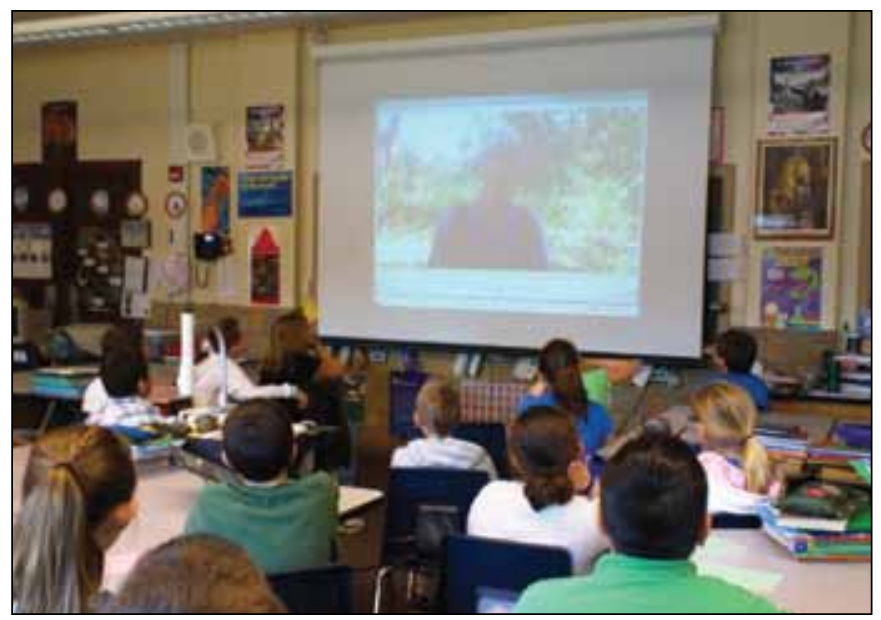

Fig. 4: Students viewing the virtual conference 


\section{Virtual Conference: Summary}

All in all, the conference was a success. The student research reports demonstrated the goals of L2R. First, a majority of the projects illustrated collaboration via technology. For example, students from Puerto Rico and Alaska worked together to compare their local climates using GLOBE protocols and other schools referenced conversations with scientists from around the world. Second, all of the student projects demonstrated authentic Earth System Science research ranging from local to global scales. The quality of the projects varied, but they all illustrated a scientific research project from hypothesis to conclusion. Additionally, the students had a common space to see similar types of research and compare their local environments to other environments around the country.

\section{Reflection}

With one year of the Virtual Student Conference complete, the L2R staff came to several realizations. First, having a one-day conference was too short. For the next virtual conference, the open window will be three weeks. (Note: The next GLOBE Virtual Student Conference takes place May 6-31, 2013 and projects will be viewable any time after May 6, 2013.) This will allow for more meaningful discussions to occur between the students, scientists, and GLOBE community. This will also allow flexibility for scheduled field trips, school activities, and testing. Second, the virtual conference will be open to the GLOBE community. Students will be able to see student research projects from other parts of the world, as well as other L2R schools. Third, rather than just limiting the comments to GLOBE scientists and L2R teachers and students, the comments will be open to the entire GLOBE community. A more diverse population viewing and commenting on the projects should lead to more interesting conversations. Finally, projects will be scored by scientists from the GLOBE International Scientist Network using a modified Intel Science Fair scoring sheet. Scoring is modified to include points for entering data into the GLOBE database, which then makes it available for students and the public to utilize this data.

As for working with the teachers, several changes were implemented in the elements of the professional development from the first to the second cohort. First, more time was spent covering the scientific research process and how to develop a researchable question during the summer workshop. Second, the new technologies were practiced repeatedly during the workshop. Before the teachers left, each had to successfully Skype, use Adobe Connect, and enter test data into the GLOBE database. 
Third, each teacher completed a project plan that included plans for collecting data, finding climate data sources, and plans for how to collaborate with other schools and scientists. GLOBE scientists and L2R staff worked through the project plans with each teacher. While the second student virtual conference has not yet happened, the teacher updates already indicate these additional steps are helping to shape more collaborative and scientifically sound research projects.

In conclusion, several aspects were found to be the most effective in helping engage students in scientific research. First, using technology and finding new ways for using technology interested the students. Second, forming connections with other students, schools and scientists made learning about distant locations more meaningful. Third, finding locally relevant and personally significant science topics helped keep the students motivated to explore their research questions. Finally, the L2R staff worked closely with the teachers to provide support and assistance. Overall, everyone involved in working with the students and teachers in some way was impressed with the students' interest and passion about climate issues.

\section{References}

Capps, D.K., Crawford, B.A., \& Constas, M.A. (2012). A review of empirical literature on inquiry professional development: Alignment with best practices and a critique of the findings. Journal of Science Teacher Education, 23(3), 291-318.

Darling-Hammond, L., \& McLaughlin, M. W. (1995). Policies that support professional development in an era of reform. Phi Delta Kappan, 76(8), 597-604.

Fleming, M. L. (2010). TEACHERS' NEEDS: Professional Development Priorities of Formal Pre-K-20 Environmental Educators (p. 26). Environmental Education and Training Partnership.

Garet, M. S., Porter, A. C., Desimone, L., Birman, B. F., \& Yoon, K. S. (2001). What makes professional development effective? Results from a national sample of teachers. American Educational Research Journal, 38(4), 915-945.

Hodson,D.(2003). Timeforaction:Scienceeducation for an alternative future. International
Journal of Science Education, 25(6), 645670. doi:10.1080/09500690305021

Lenhart, A., Purcell, K., Smith, A., \& Zickuhr, K. (2010). Social media \& mobile internet use among teens and young adults. Washington, DC: Pew Internet \& American Life Project.

Loucks-Horsley, S., Hewson, P. W., Love, N., \& Stiles, K. E. (1998). Designing professional development for teachers of science and mathematics. Thousand Oaks, CA: Corwin Press.

National Research Council. (2012). A Framework for K-12 Science Education: Practices, Crosscutting Concepts, and Core Ideas. National Academies Press.

Penuel, W. R., Fishman, B. J., Yamaguchi, R., \& Gallagher, L. P. (2007). What makes professional development effective? Strategies that foster curriculum implementation. American Educational Research Journal, 44(4), 921-958. 


\section{Acknowledgments and Disclaimer}

"This material is based upon work supported by the National Science Foundation under Grant No. 0929725. Any opinions, findings and conclusions or recommendations expressed in this material are those of the author(s) and do not necessarily reflect the views of the National Science Foundation (NSF)."

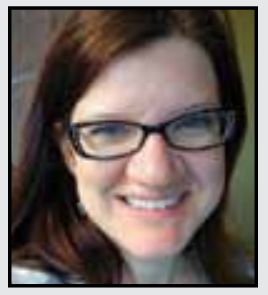

Julie S. Malmberg is a Project Manager and Principal Investigator with The GLOBE Program in Boulder, CO. She works with schools located around the world to investigate local climates and to study the Earth System as a whole. Julie has a BS and MS in Atmospheric Sciences from the University of Illinois at Urbana-Champaign and is currently a PhD candidate in Geography at the University of Colorado at Boulder. Much of Julie's research has focused on education and earth sciences. Julie is also an adjunct meteorology professor at an online university and works to make science more accessible to non-science majors.

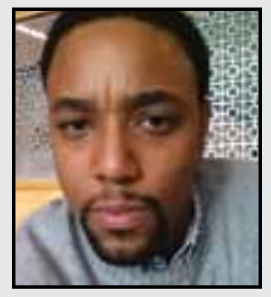

Keith E. Maull is a PhD candidate in the Department of Computer Science at the University of Colorado at Boulder. His research focuses on educational informatics, with particular interests in the role data analytics play in improving awareness, effectiveness and outcomes through technology platforms for educators and students in STEM disciplines. His professional background includes extensive software development experience from Fortune 50 companies to startups and non-profit organizations.

LINK TO:

http://www.colorado.edu 